\title{
High-Wind Drag Coefficient and Whitecap Coverage Derived from Microwave Radiometer Observations in Tropical Cyclones
}

\author{
PAUL A. HWANG \\ Remote Sensing Division, Naval Research Laboratory, Washington, D.C.
}

(Manuscript received 29 May 2018, in final form 27 July 2018)

\begin{abstract}
Ocean surface roughness and whitecaps are driven by the ocean surface wind stress; thus, their values calculated from the wind speed input may vary significantly depending on the drag coefficient formula applied. Because roughness and whitecaps are critical elements of the ocean surface response in microwave remote sensing, the extensive microwave remote sensing measurements contain the information of the drag coefficient, surface roughness, and whitecap coverage. The scattering radar cross sections from global measurements under calm to tropical cyclone conditions have been used effectively to improve the formulation of the surface roughness spectrum. In this paper, the microwave radiometer measurements in tropical cyclones are exploited to extract information of the drag coefficient and whitecap coverage in high winds. The results show that when expressed as a wind speed power function, the exponent in high winds (greater than about $35 \mathrm{~m} \mathrm{~s}^{-1}$ ) is about -1 for the drag coefficient, 0.5 for the wind friction velocity, and 1.25 for the whitecap coverage.
\end{abstract}

\section{Introduction}

Ocean surface wind stress and surface wave breaking are among the most fascinating upper-ocean properties that have significant impacts on many physical oceanographic processes. Direct measurements of wind stress and wave breaking are rare. In practice, the wind stress is computed with wind velocity using various parametric formulas for the drag coefficient. Many experiments have been undertaken to refine the drag coefficient equations, especially to extend the wind speed range into tropical cyclone (TC) conditions (e.g., Powell et al. 2003; Jarosz et al. 2007; Bell et al. 2012; Holthuijsen et al. 2012). For wave breaking, whitecaps are among the most convenient observables. Although there were countless reported experiments, the highest wind speed remains about $25 \mathrm{~m} \mathrm{~s}^{-1}$; for example, see the recent review by Brumer et al. (2017).

Microwave remote sensing plays an important role in physical oceanography research, ranging from ocean circulations from altimeter sea surface height measurements to global ocean surface vector wind fields from active and passive microwave sensors including altimeter, scatterometer, radiometer, and hybrid systems combining passive receivers and existing active spaceborne

Corresponding author: Dr. Paul A. Hwang, paul.hwang@nrl. navy.mil microwave sources from the global positioning system (GPS) or other communication satellites. The received microwave signals contain the signatures of different oceanographic processes that modify the electromagnetic (EM) emission or scattering to the receivers. In particular, ocean surface roughness and whitecap coverage are two main contributors to the received signals in microwave wind sensing. Because both surface roughness and whitecaps are driven by the surface wind stress, these microwave signals are a good source for investigating the drag coefficient. Sorting out the complex relationships between the microwave signals, the drag coefficient, the ocean surface roughness, and the whitecap coverage, however, is not trivial.

Scatterometers operating in moderate incidence angles (approximated between $30^{\circ}$ and $60^{\circ}$ ) serve as the ocean surface roughness spectrometer because of the dominance of the Bragg resonance scattering mechanism (e.g., Wright 1966, 1968; Plant 1990; and references therein). The Bragg resonance attribute has been used to refine the ocean surface roughness spectral model (Hwang et al. 2013; Hwang and Fois 2015) using the L-, C-, and Ku-band (approximately 1.4, 5.3, and $14 \mathrm{GHz}$ ) geophysical model functions (GMFs), which are established from long-term global wind-sensing efforts relating the wind velocity and the backscattering normalized radar cross section (NRCS); the wind speed range is from calm to TC conditions. More recently, the low-pass-filtered mean-square slope 
(LPMSS) observations in hurricanes using GPS reflectometry (Katzberg and Dunion 2009; Gleason 2013; Katzberg et al. 2013; Gleason et al. 2018) were used to further refine the wave spectrum model near the dominant wavelength scales (Hwang and Fan 2018).

The subject of the surface roughness spectrum has been described in great detail in the papers cited in the last paragraph and the references therein. The present investigation focuses on the drag coefficient and whitecap coverage. In particular, the microwave radiometer signals are exploited for recovering the whitecap information, as well as the associated drag coefficient properties. Radiometers measure the sea surface brightness temperature $T_{B}$, for which the surface roughness and whitecap coverage make strong contributions but through different mechanisms. The surface roughness contribution can be calculated with the ocean polarimetric thermal emission formulation (e.g., Yueh et al. 1994a,b; Johnson and Zhang 1999). The whitecap contribution can be evaluated with the modification of the water-side relative permittivity considering the entrained air from wave breaking, which leads to the change of air-sea interface emission and scattering properties (Stogryn 1972, hereafter S72; Reul and Chapron 2003; Hwang 2012, hereafter H12; Hwang and Fois 2015). A brief summary of the mathematical formulation is given in section 2 .

Of special interest for the analysis of whitecap coverage and the related drag coefficient formulation are the measurements derived from the C-band (4.74$7.09 \mathrm{GHz}$ ) Stepped Frequency Microwave Radiometer (SFMR) obtained in hurricane reconnaissance and research missions with wind speed exceeding $70 \mathrm{~m} \mathrm{~s}^{-1}$ (e.g., Uhlhorn and Black 2003; Uhlhorn et al. 2007; Klotz and Uhlhorn 2014, hereafter K14). The results of the drag coefficient and whitecap coverage derived from the SFMR observations are given in section 3. Section 4 presents discussions on the frequency dependence, foam and roughness contributions, and the drag coefficient and whitecap observations. Section 5 is a summary.

\section{Roughness and whitecap contributions to microwave brightness temperature}

\section{a. Formulation}

The brightness temperature $T_{B}$ of horizontal $(\mathrm{H})$ or vertical $(\mathrm{V})$ polarization at microwave frequency $f$, incidence angle $\theta$, and azimuth angle $\phi$ (with respect to the wind direction) can be expressed as (Yueh et al. 1994a,b; Johnson and Zhang 1999; H12)

$$
\begin{aligned}
{\left[\begin{array}{c}
T_{B \mathrm{H}}(f, \theta, \phi) \\
T_{B \mathrm{~V}}(f, \theta, \phi)
\end{array}\right] } & =T_{s}\left(\begin{array}{c}
e_{\mathrm{H}} \\
e_{\mathrm{V}}
\end{array}\right) \\
& =T_{s}\left[\left(\begin{array}{c}
e_{0 \mathrm{H}} \\
e_{0 \mathrm{~V}}
\end{array}\right)+\left(\begin{array}{c}
\Delta e_{f \mathrm{H}} \\
\Delta e_{f \mathrm{~V}}
\end{array}\right)+\left(\begin{array}{c}
\Delta e_{r \mathrm{H}} \\
\Delta e_{r \mathrm{~V}}
\end{array}\right)\right],
\end{aligned}
$$

where $T_{s}$ is the sea surface temperature and $e_{p}(f, \theta, \phi)$ is the sea surface emissivity of $p$ polarization. The emissivity can be decomposed into three parts, as shown in the last equality in (1). The first term represents the contribution from a foamless flat surface:

$$
e_{0 p}(f, \theta)=1-\left|R_{p p}^{(0)}(f, \theta)\right|^{2} .
$$

The variable $R_{p p}^{(0)}(f, \theta)$ is the Fresnel reflection coefficient, which is a function of the frequency-dependent relativity permittivity $\varepsilon$ and $\theta$ :

$$
\begin{aligned}
& R_{\mathrm{HH}}^{(0)}=\frac{\left[\cos \theta-\left(\varepsilon-\sin ^{2} \theta\right)^{1 / 2}\right]^{2}}{\left[\cos \theta+\left(\varepsilon-\sin ^{2} \theta\right)^{1 / 2}\right]^{2}}, \\
& R_{\mathrm{VV}}^{(0)}=\frac{\left[\varepsilon \cos \theta-\left(\varepsilon-\sin ^{2} \theta\right)^{1 / 2}\right]^{2}}{\left[\varepsilon \cos \theta+\left(\varepsilon-\sin ^{2} \theta\right)^{1 / 2}\right]^{2}} .
\end{aligned}
$$

There are several different formulas for the relative permittivity of seawater (e.g., Klein and Swift 1977; Meissner and Wentz 2004). They produce only minor differences for the C-band frequencies of concern here. The formulation by Meissner and Wentz (2004) is used in this paper [their (7), (8), and (12)-(17)]. Throughout this paper, the values 35 psu and $301 \mathrm{~K}$ $\left(28^{\circ} \mathrm{C}\right)$ are used for the required input of seawater salinity and temperature for the computation of the relative permittivity.

The two wind-induced terms $\Delta e_{f p}(f, \theta, \phi)$ and $\Delta e_{r p}(f, \theta, \phi)$ are the emissivity changes due to foam (whitecaps) and the rough sea surface, respectively. These two terms contain the information of the geophysical parameters embedded in the radiometer signals; of interest in this paper are the wind speed, wind stress, surface roughness, and whitecap coverage.

Extensive research has been devoted to the windinduced roughness term $\Delta e_{r p}$ (e.g., Yueh et al. 1994a,b; Johnson and Zhang 1999). Briefly, it is given as

$$
\left[\begin{array}{l}
\Delta e_{r \mathrm{H}}(f, \theta, \phi) \\
\Delta e_{r \mathrm{~V}}(f, \theta, \phi)
\end{array}\right]=\int_{0}^{\infty} \int_{0}^{2 \pi} F\left(k^{\prime}, \phi^{\prime}\right)\left[\begin{array}{l}
g_{\mathrm{H}}\left(f, \theta, \phi, \varepsilon, k^{\prime}, \phi^{\prime}\right) \\
g_{\mathrm{V}}\left(f, \theta, \phi, \varepsilon, k^{\prime}, \phi^{\prime}\right)
\end{array}\right] k^{\prime} d \phi^{\prime} d k^{\prime}
$$


where $\varepsilon$ is the water-side relative permittivity, $g_{p}$ is the EM weighting function describing the contribution of each wavenumber-directional surface wave component to the thermal emission [the full expression of the $g_{p}$ term is given in Yueh et al. (1994a,b) and Johnson and Zhang (1999)], $F$ is the surface wave spectrum (the ocean surface roughness), and $k$ is the wavenumber. In Yueh et al. (1994a,b) and Johnson and Zhang (1999), the (foamless) seawater relative permittivity $\varepsilon_{\mathrm{sw}}$ is used for $\varepsilon$, and whitecap contribution is not treated explicitly in their formulation.

To quantify the foam effect from the air in whitecaps, H12 considers an effective relative permittivity $\varepsilon_{e}$ of the air-water mixture, which is computed with the quadratic mixing rule in a similar fashion as discussed in Anguelova (2008):

$$
\varepsilon_{e}=\left[F_{a} \varepsilon_{a}^{1 / 2}+\left(1-F_{a}\right) \varepsilon_{\mathrm{sw}}^{1 / 2}\right]^{2},
$$

where $\varepsilon_{a}=1$ is the relative permittivity of air, $\varepsilon_{\mathrm{sw}}$ is the foamless seawater relative permittivity as mentioned earlier, and $F_{a}$ is the air fraction.

The $\Delta e_{f p}$ term is defined as the difference between the foamless flat surface emissivity and the foamed flat surface emissivity. For the former, the Fresnel reflection coefficient in (3) is calculated with $\varepsilon=\varepsilon_{\mathrm{sw}}$, and for the latter, $\varepsilon=\varepsilon_{e}$ is employed. For simplicity's sake, the terms roughness and foam contributions are used for $\Delta e_{r p}$ and $\Delta e_{f p}$, respectively; although strictly speaking, the foam effect is also in the $\Delta e_{r p}$ term because $\varepsilon=\varepsilon_{e}$ is used in (4).

\section{b. Implementation}

When surface waves break, they frequently entrain air into the water column. Because the relative permittivities of air and water differ significantly, even a small amount of air can produce a large change of the relative permittivity of the resulting mixture. To quantify the effects of entrained air, ideally, we need to know the fraction of air in water (void fraction) and the evolving size distribution and entrainment depth of the bubble clouds that carry the air into water. Such oceanographic knowledge is sorely inadequate. However, it has been verified that the vertical distribution of bubble clouds or void fraction decays rapidly with distance from the airsea interface, so the majority of the penetrated air is near the sea surface.

A rigorous treatment of microwave interaction with surface foam requires detailed oceanographic information of the bubble cloud spatial and size distributions. Such information is not available currently. $\mathrm{H} 12$ uses the whitecap fraction $W_{c}$ in place of the void fraction $F_{a}$ in the mixing rule for the evaluation of the effective relative permittivity $\varepsilon_{e}$ in (5). Considering the shallow penetration depth of microwaves, for example, the skin depth is about $2 \mathrm{~mm}$ at $10 \mathrm{GHz}$ and $5 \mathrm{~mm}$ at $5 \mathrm{GHz}$ (Plant 1990), this approximation seems to be reasonable. The whitecap coverage represents an upper bound of the void fraction because it is equivalent to assuming $100 \%$ of air in the depth of microwave influence under the foamy area, whereas the actual air entrainment decreases exponentially with water depth.

Following the analysis presented in $\mathrm{H} 12$ and with the consideration that the surface wind stress drives the upper-ocean processes, the formula for the whitecap coverage $W_{c}$ is parameterized with the friction velocity $u_{*}$ using the whitecap data reported in Callaghan et al. (2008):

$$
W_{c}=\left\{\begin{array}{cl}
0, & u_{*} \leq 0.11 \mathrm{~m} \mathrm{~s}^{-1} \\
0.30\left(u_{*}-0.11\right)^{3}, & 0.11<u_{*} \leq 0.40 \mathrm{~m} \mathrm{~s}^{-1} \\
0.07 u_{*}^{2.5}, & u_{*}>0.40 \mathrm{~m} \mathrm{~s}^{-1}
\end{array}\right.
$$

The drag coefficient used for computing $u_{*}$ from $U_{10}$, that is, $C_{10}=u_{*}^{2} U_{10}^{-2}$, is by least squares fitting of three open-ocean datasets (Felizardo and Melville 1995; Powell et al. 2003; Jarosz et al. 2007),

$$
C_{10 A}=10^{-4}\left(-0.0160 U_{10}^{2}+0.967 U_{10}+8.058\right) .
$$

The formula (7) captures the saturation and decaying behavior of the drag coefficient in high winds; the computed wind friction velocity $u_{*}$ increases monotonically with $U_{10}$ up to $50 \mathrm{~m} \mathrm{~s}^{-1}$ and then decreases for $U_{10}>$ $50 \mathrm{~m} \mathrm{~s}^{-1}$ [see Fig. 1 of Hwang et al. (2013) and Fig. 3b in this paper to be discussed later]. The $C_{10}$ formula in (7) was originally given by Hwang (2011) and suggested to be applicable for $U_{10} \leq 50 \mathrm{~m} \mathrm{~s}^{-1}$. It is recognized that there is still considerable uncertainty in the ocean surface drag coefficient in very high winds, and indeed, the analysis of SFMR data collected in TCs, to be presented in section 3, provides the important (indirect) field measurements to improve the drag coefficient formulation (section $3 b$ ).

\section{c. Verification}

The emissivity calculated by $1-\left|R_{p p}^{(0)}\right|^{2}$ with the "equivalent medium" relative permittivity in (5) produces similar results as that using the mixing rule applied to the emissivity of foamless sea surface area and the emissivity of $100 \%$ foam area as treated in S72 for the horizontal polarization, but the two solutions differ significantly for the vertical polarization in high winds. Figure 1 shows the C-band $(4.74 \mathrm{GHz})$ results computed with $U_{10}$ in $3 \mathrm{~m} \mathrm{~s}^{-1}$ steps. For the foamless flat surface 


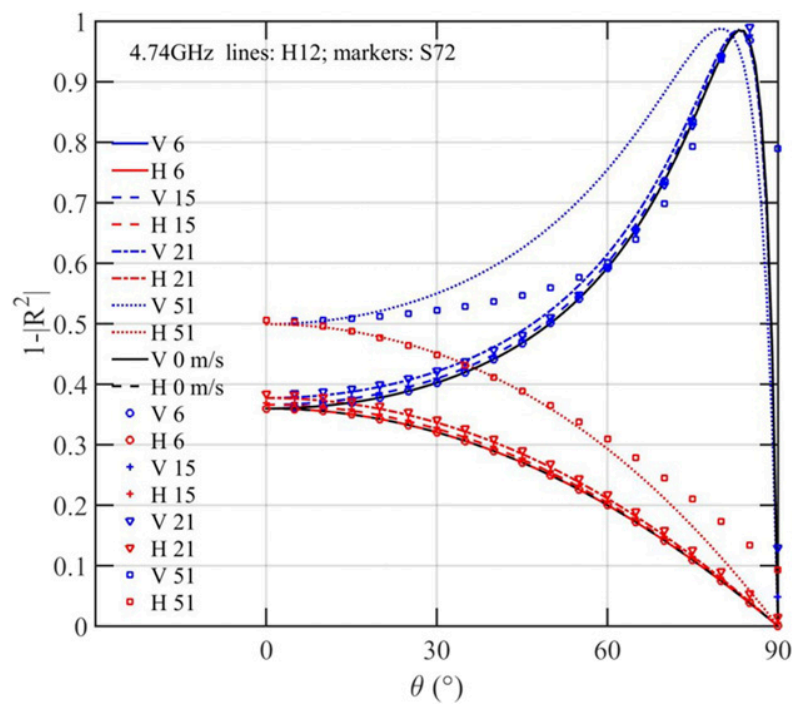

FIG. 1. Comparison of the emissivity of a flat surface covered with foam from wave breaking computed with the emissivity mixing formula by S72 (markers) and the permittivity mixing approach by $\mathrm{H} 12$ (lines).

reference, the black solid and dashed lines show the vertical and horizontal polarizations, respectively. For the wind-disturbed surfaces, the vertical and horizontal polarizations are illustrated with blue and red colors, respectively; smooth lines are for the $\mathrm{H} 12$ algorithm, and markers are for the S72 algorithm. The foam effect is relatively small for $U_{10}$ less than or equal to $15 \mathrm{~m} \mathrm{~s}^{-1}$ and becomes rather substantial in very high winds. For clarity, only the results for $6,15,21$, and $51 \mathrm{~m} \mathrm{~s}^{-1}$ are displayed; the $6 \mathrm{~m} \mathrm{~s}^{-1}$ results are indistinguishable from the flat surface reference in the illustration.

There is a fundamental difference between the S72 and $\mathrm{H} 12$ approaches treating the modification of the water-side dielectric property from air entrainment in the whitecaps. S72 applies the mixing to the observed emissivities in several field and laboratory experiments (their Table 1). Polynomial equations are then developed to interpolate or extrapolate in the frequency and incidence angle ranges. H12 applies the mixing to the relative permittivities of seawater and air weighted with the whitecap fraction as described in section $2 \mathrm{~b}$; the emissivity is then computed with the effective (equivalent medium) permittivity.

The calculated brightness temperature as a function of wind speed based on the equivalent medium approach is in very good agreement with a global dataset of WindSat microwave radiometer measurements with wind speed coverage up to about $42 \mathrm{~m} \mathrm{~s}^{-1}$ (Meissner and Wentz 2009). The WindSat measurements include five microwave frequencies $(6,10,18,23$, and $37 \mathrm{GHz})$ for both vertical and horizontal polarizations.
The wind disturbance on the sea surface causes changes of the measured brightness temperature from the flat surface reference values. These changes are given as an excess emissivity, which is then used to derive the wind velocity from the brightness temperature observation. The WindSat data (measured at the $53^{\circ}$ incidence angle) show the trend of increasing excess emissivity with wind speed, and the magnitude of the excess emissivity is larger for the horizontal polarization than the vertical polarization. Furthermore, there is a general increasing trend of the excess emissivity with microwave frequency for both vertical and horizontal polarizations; see Fig. 1 in Meissner and Wentz (2009) or Fig. 3 in H12.

The surface roughness spectrum model, which impacts the resulting emission or scattering computation, has gone through a couple of major revisions. For each revision, the high-wind passive microwave radiometer dataset by Meissner and Wentz (2009), together with other active NRCS data sources, has been used to verify the improved performance of the revised roughness spectral model, as shown in Fig. 9 of Hwang et al. (2013) and Fig. 5 of Hwang and Fois (2015).

\section{SFMR brightness temperature, drag coefficient, and whitecap coverage}

\section{a. SFMR observations in tropical cyclones}

The SFMR is the most successful instrument for obtaining sea surface wind speed and rain rate simultaneously inside TCs. Extensive discussions on the SFMR operations and data processing have been published, for example, Uhlhorn and Black (2003), Uhlhorn et al. (2007), and K14 and references therein. Here, the discussion is limited to the application of using the SFMR brightness temperature to retrieve the information of whitecaps and drag coefficient. Briefly, the SFMR is an airborne radiometer that operates at six closely spaced C-band channels in the range of 4.6-7.2 GHz. For hurricane reconnaissance and research missions, it obtains the sea surface brightness temperature along the aircraft flight track in the nadirlooking configuration, for which the results from the horizontal and vertical polarizations are identical.

K14 report a comprehensive analysis of the SFMR measurements from 370 hurricane reconnaissance and research missions: 200 missions from U.S. Air Force Reserve Command (AFRC) and 170 missions from National Oceanic and Atmospheric Administration (NOAA) hurricane-penetrating aircraft. Figure 2a reproduces the left column of their Fig. 7 showing the SFMR $T_{B}$ and the derived rain absorption coefficient $\kappa$ from all six frequencies for two radial passes (inbound to and outbound from the center) in Hurricane Rita between 1506 and 1536 UTC 21 September 2005. 


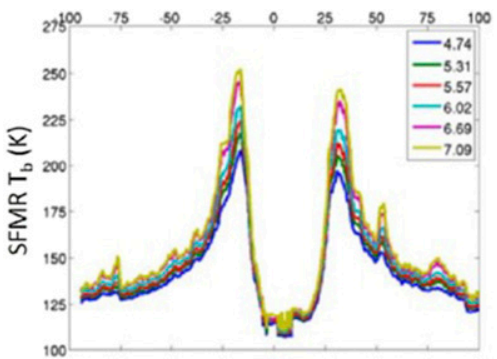

(a)

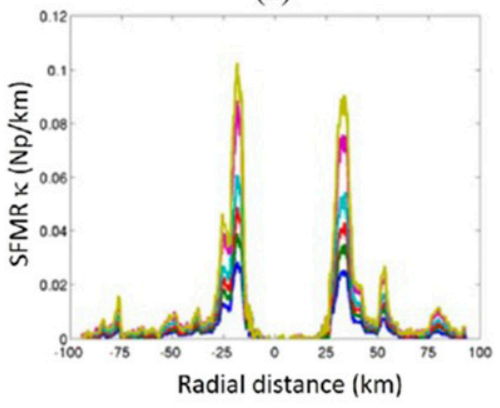

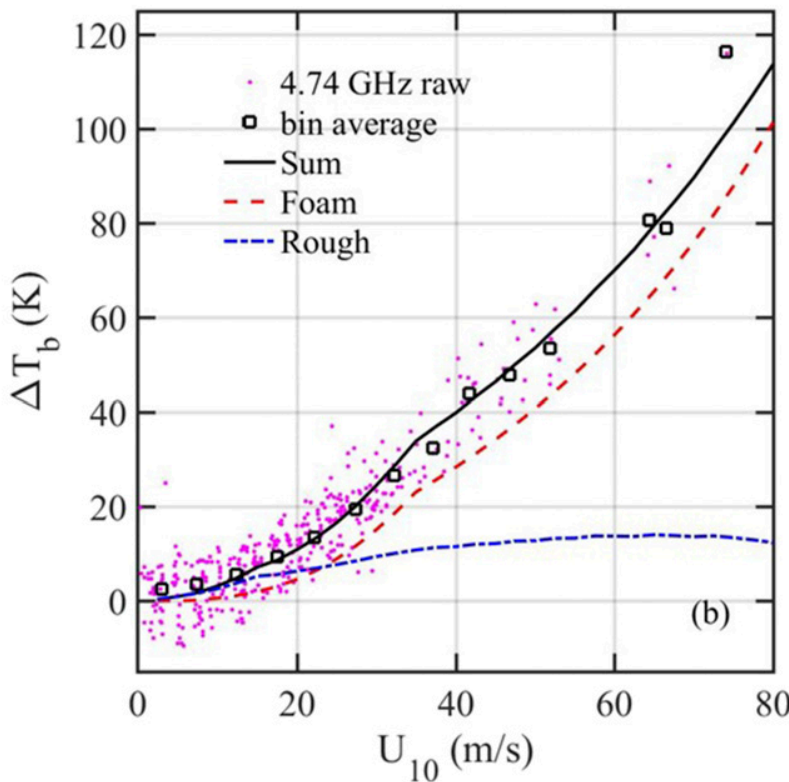

FIG. 2. (a) An example of the SFMR $T_{B}$ and the derived rain absorption coefficient $\kappa$ from all six frequencies for two radial passes (inbound to and outbound from the center) in Hurricane Rita between 1506 and 1536 UTC 21 Sep 2005, reproducing the left column of Fig. 7 of K14. (b) The 4.74-GHz $\Delta T_{B}$ as a function of the surface wind speed from dropsonde analysis, digitized from Fig. 3a of K14. The raw data are given in magenta dots, and the binaveraged results are shown with black squares. Superimposed in Fig. $3 \mathrm{~b}$ are the computed $\Delta T_{B}$ sum, roughness, and foam contributions using (8) for the drag coefficient.

The brightness temperature received at the sensor antenna is first processed to obtain the brightness temperature at the sea surface by correcting for the atmospheric emissions and cosmic microwave background radiation. After the attenuation by rain has been removed, the excess emissivity attributing to wind effects (both roughness and whitecaps) is reported in their Fig. 3. The data in Fig. $2 b$ are digitized from their Fig. 3a, showing the $4.74-\mathrm{GHz}$ excess emissivity as a function of the surface wind speed from dropsonde analysis; magenta dots are raw data, and black squares are the bin-averaged results. Here, the excess emissivity is expressed as the excess brightness temperature $\Delta T_{B}$ with sea surface temperature $T_{s}=301 \mathrm{~K}$.

These $\Delta T_{B}$ data can be used to investigate the drag coefficient, surface roughness spectrum, and whitecap coverage using the thermal emission model described in section 2. Because both the surface roughness spectrum and the whitecap coverage are formulated with wind stress as the driving force, the numerical experiment is primarily conducted in modifying the drag coefficient in (7), particularly in the high-wind region, with the recognition that it is where the main uncertainty resides. The detail is further described in section $3 b$.

The solid black line superimposed in Fig. $2 \mathrm{~b}$ is the final result of the computed $\Delta T_{B}$ from this research. It is in excellent agreement with the SFMR measurements; the revised drag coefficient in (8) used for the computation will be further discussed in section $3 \mathrm{~b}$. For reference, the foam and roughness contributions are also shown with red dashed and blue dashed-dotted lines, respectively. Notably, the roughness contribution dominates over a rather wide wind speed range of pre-TC conditions. It is not until $U_{10}$ exceeds about $22 \mathrm{~m} \mathrm{~s}^{-1}$ that the foam contribution becomes larger than the roughness contribution. The issue of roughness and foam contributions will be further discussed in section $4 \mathrm{~b}$.

\section{b. Drag coefficient and whitecap coverage}

Figure 3a shows several sets of $C_{10}$ field data collected in open-ocean conditions with different colors: blue (Felizardo and Melville 1995; Powell et al. 2003; Jarosz et al. 2007), red (Holthuijsen et al. 2012), green (Powell 2006), and cyan (Bell et al. 2012); except for those blue data with $U_{10}<15 \mathrm{~m} \mathrm{~s}^{-1}$, the measurements were collected inside hurricanes with different markers showing the locations of measurements. More details are given in section 4c of Hwang and Fan (2017).

The drag coefficient data can also be presented in terms of $u_{*}\left(U_{10}\right)$ as shown in Fig. 3b. The friction velocity $u_{*}$ is used in the parametric functions of the surface roughness spectrum (Hwang and Fois 2015) and the whitecap coverage $W_{c}$ in (6). Figure $3 c$ shows the 

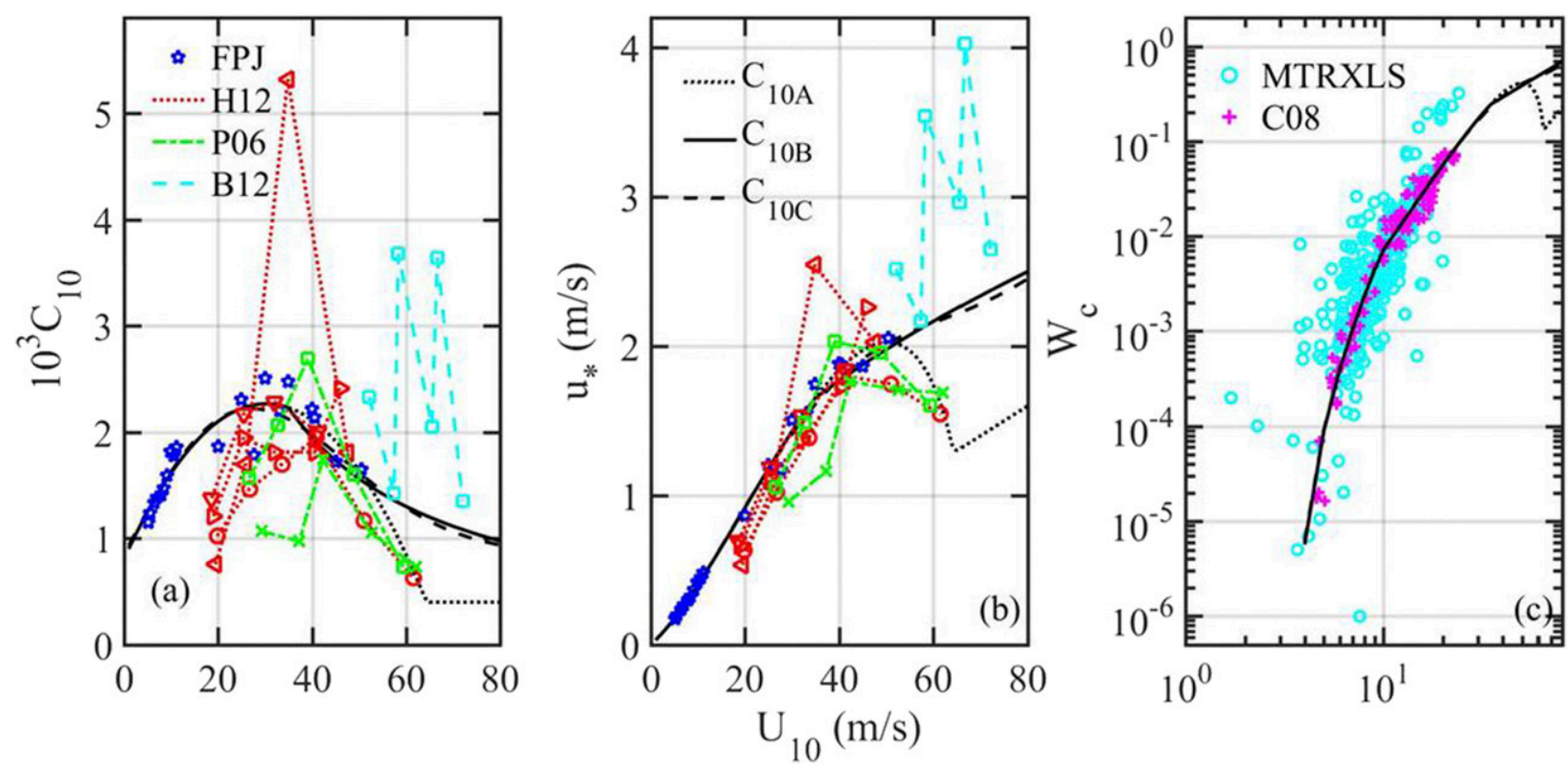

FIG. 3. (a) The $C_{10}$ data collected in open-ocean conditions with different colors: blue (Felizardo and Melville 1995; Powell et al. 2003; Jarosz et al. 2007), red (Holthuijsen et al. 2012), green (Powell 2006), and cyan (Bell et al. 2012); except for those blue data with $U_{10}<$ $15 \mathrm{~m} \mathrm{~s}^{-1}$, the measurements were collected inside hurricanes with different markers showing the locations of measurements. More details are given in Hwang and Fan (2017). (b) The drag coefficient expressed in terms of the friction velocity $u *$. (c) The whitecap measurements reported by Monahan (1971), Toba and Chaen (1973), Ross and Cardone (1974), Xu et al. (2000), Lafon et al. (2004, 2007), Sugihara et al. (2007), and Callaghan et al. (2008); the first six sources are collectively labeled as the MTRXLS dataset (Hwang and Sletten 2008) in the figure legend. The three drag coefficient formulas, (7)-(9), discussed in this paper and the resulting $u *$ and $W_{c}$ are shown with black lines (dotted, solid, and dashed, respectively).

whitecap measurements reported by Monahan (1971), Toba and Chaen (1973), Ross and Cardone (1974), Xu et al. (2000), Lafon et al. (2004, 2007), Sugihara et al. (2007), and Callaghan et al. (2008); the first six sources are collectively labeled as the MTRXLS dataset (Hwang and Sletten 2008) in the figure legend. The outlines of these collective data are the typical envelopes of the field measurements; for example, compare the results with Fig. 9a of Brumer et al. (2017).

Callaghan et al. (2008) analyze 43158 video images using an automated whitecap extraction algorithm; each image covers an area of about $4997 \mathrm{~m}^{2}$. Their analysis yields $107 W_{c}$ data points with wind speed coverage between 3.7 and $23.1 \mathrm{~m} \mathrm{~s}^{-1}$. Their data, shown with magenta pluses in Fig. $3 \mathrm{c}$ going through the center of the MTRXLS data cloud, were used as the basis of developing the parametric whitecap formula in (6) by H12.

As noted in section $2 \mathrm{~b}$, the $C_{10}$ formula in (7), shown with the black dotted line in Fig. 3a, was based on least squares fitting through the blue set of the deep-ocean data (denoted as FPJ in the legend), and it was suggested to be applicable for $U_{10} \leq 50 \mathrm{~m} \mathrm{~s}^{-1}$ (Hwang 2011). This is the first formula tested for analyzing the SFMR data discussed in section 3a. To prevent negative value in the computed $C_{10}$ for using (7) beyond $U_{10}=50 \mathrm{~m} \mathrm{~s}^{-1}$, an arbitrary minimum value of $C_{10 \mathrm{~min}}=4 \times 10^{-4}$ is assigned; this $C_{10 \min }$ applies to the wind speed range $U_{10} \geq 64.4 \mathrm{~m} \mathrm{~s}^{-1}$.

The computed $\Delta T_{B}$ is given with dotted lines in Fig. 4a. The $T_{B}$ computation using the drag coefficient formula in (7) is obviously not good for $U_{10}$ greater than about $51 \mathrm{~m} \mathrm{~s}^{-1}$. The result indicates that the drag coefficient formula in the very high-wind region requires some alteration. A general functional form of the drag coefficient in the high-wind branch $U_{10}>U_{10 t}$, $C_{10 h}=C_{10 t}\left(U_{10} / U_{10 t}\right)^{a_{t}}$, is examined with various combinations of $U_{10 t}$ and $a_{t}$ constrained by $U_{10 t} \leq 50 \mathrm{~m} \mathrm{~s}^{-1}$ and $a_{t} \leq 0\left[C_{10 t}\right.$ is the drag coefficient of $U_{10 t}$ calculated by (7)]. The goal is to match the computed $T_{B}$ with the SFMR data.

The following formula produces the good agreement shown in Fig. 2b:

$$
C_{10 B}=\left\{\begin{array}{cc}
10^{-4}\left(-0.0160 U_{10}^{2}+0.967 U_{10}+8.058\right), & U_{10} \leq 35 \mathrm{~m} \mathrm{~s}^{-1} \\
2.23 \times 10^{-3}\left(U_{10} / 35\right)^{-1}, & U_{10}>35 \mathrm{~m} \mathrm{~s}^{-1}
\end{array}\right.
$$




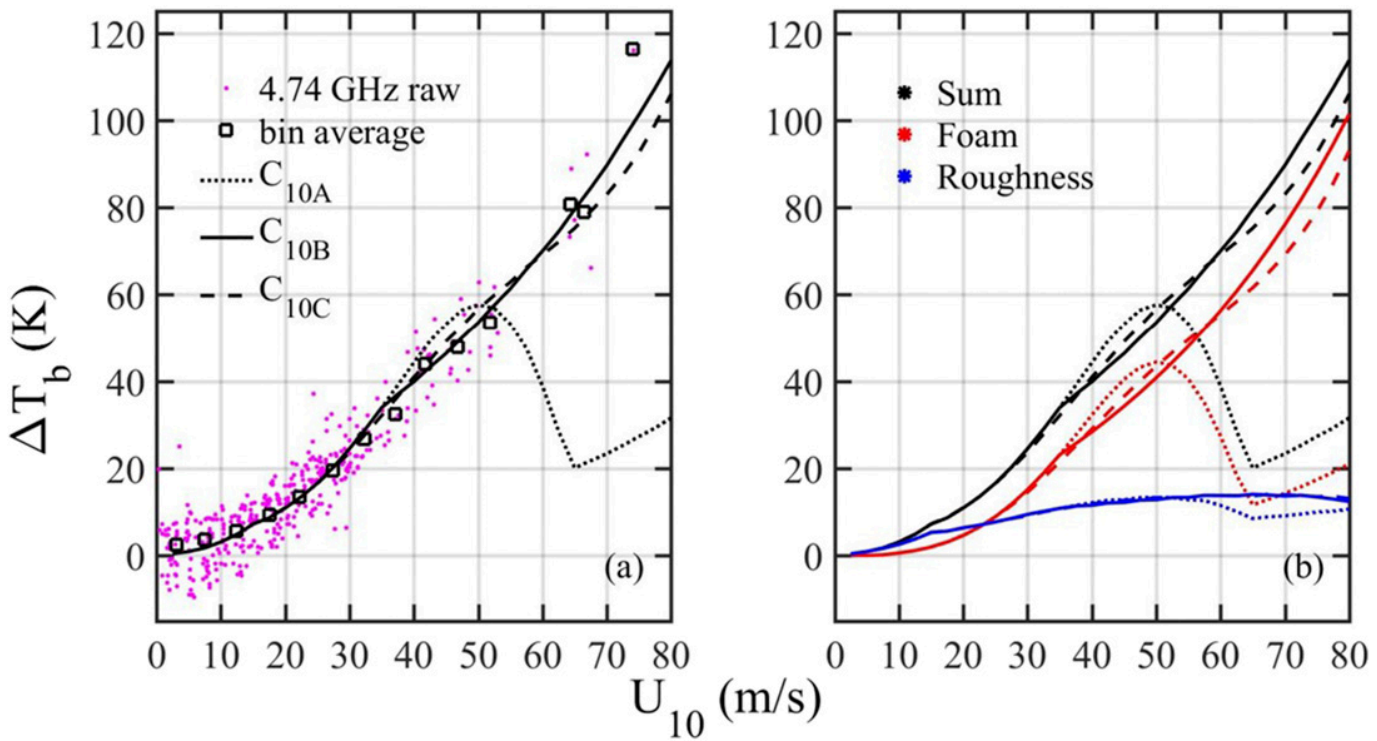

FIG. 4. (a) The 4.74-GHz $\Delta T_{B}$ computed with different drag coefficient formulas and comparison with SFMR measurements. The results computed with $C_{10 A}, C_{10 B}$, and $C_{10 C}$ formulas are shown with dotted, solid, and dashed lines, respectively. (b) The sum, foam, and roughness contributions of the 4.74-GHz $\Delta T_{B}$ are shown separately with black, red, and blue curves, respectively; the different line styles represent different drag coefficient formulas as in (a).

In Figs. 3 and 4, solid lines show the computation results of $C_{10 B}, u_{*}, W_{c}$, and $\Delta T_{B}$ (sum, foam, and roughness contributions).

The $T_{B}$ computation is sensitive to the $C_{10}$ formulation. In an attempt to smooth out the kink of the computed $T_{B}$ curve at $35 \mathrm{~m} \mathrm{~s}^{-1}$ caused by the two-branch formulation in (8), a Rayleigh-like function is derived from least squares fitting to approximate (8) computed for $U_{10}$ between 0 and $80 \mathrm{~m} \mathrm{~s}^{-1}$ :

$$
C_{10 C}=a_{0}+a_{1}\left(\frac{U_{10}}{a_{2}^{2} U_{\text {ref }}}\right) \exp \left[\frac{-\left(U_{10} / U_{\text {ref }}\right)^{2}}{2 a_{2}^{2}}\right],
$$

where $U_{\text {ref }}=65 \mathrm{~m} \mathrm{~s}^{-1}, a_{0}=8.50 \times 10^{-4}, a_{1}=9.48 \times 10^{-4}$, and $a_{2}=0.419$. Dashed lines in Figs. 3 and 4 show the $C_{10 C}, u_{*}, W_{c}$, and the $\Delta T_{B}$ computation results. Although the values of $C_{10}, u_{*}$, and $W_{c}$ calculated by $C_{10 B}$ and $C_{10 C}$ are very similar (Fig. 3 , solid and dashed lines), the $\Delta T_{B}$ dependence on wind speed differ obviously in various wind speed ranges, with 4-8.5-K difference for $U_{10}$ greater than $65 \mathrm{~m} \mathrm{~s}^{-1}$ (Fig. 4).

The $\Delta T_{B}$ difference between using $C_{10 B}$ and $C_{10 C}$ is almost entirely from the foam contribution. Figure $4 \mathrm{~b}$ shows the computed results for the sum, foam, and roughness contributions with black, red, and blue lines, respectively. The roughness contributions computed with $C_{10 B}$ and $C_{10 C}$ (solid and dashed lines, respectively) are essentially indistinguishable. Further discussion on the foam and roughness contributions is given in section $4 \mathrm{~b}$.

Based on the analysis presented above, it is concluded that in very high winds ( $U_{10}$ greater than about $35 \mathrm{~m} \mathrm{~s}^{-1}$ ), $C_{10} \sim U_{10}^{-1}, u_{*} \sim U_{10}^{0.5}$, and $W_{c} \sim U_{10}^{1.25}$. The drag coefficient formula in (8) yields slightly better agreement between the thermal emission model computation and the SFMR measurements than (9), and the whitecap formula in (6) is applicable for the wide range of wind speeds encountered in the SFMR measurements.

\section{Discussion}

\section{a. Multiple-frequency applications}

As mentioned earlier, the thermal emission model (section 2) has been applied to a high-wind dataset of global WindSat analysis by Meissner and Wentz (2009). The maximum wind speed is about $42 \mathrm{~m} \mathrm{~s}^{-1}$. The WindSat measurements include five microwave frequencies $(6,10$, 18,23 , and $37 \mathrm{GHz}$ ) for both vertical and horizontal polarizations, and the nominal incidence angle is $53^{\circ}$. The observed range is from -1.1 to $24.9 \mathrm{~K}$ for $\Delta T_{\mathrm{V}}$ and from 9.7 to $63.5 \mathrm{~K}$ for $\Delta T_{\mathrm{H}}$. With successive improvements of the roughness spectrum, the model-measurement comparison statistics combining the vertical and horizontal polarizations reported by Hwang and Fois (2015) are bias of $0.74 \mathrm{~K}$, slope of linear regression of 1.04, root-mean-square (RMS) difference of $4.46 \mathrm{~K}$, and correlation coefficient of 0.96 . 

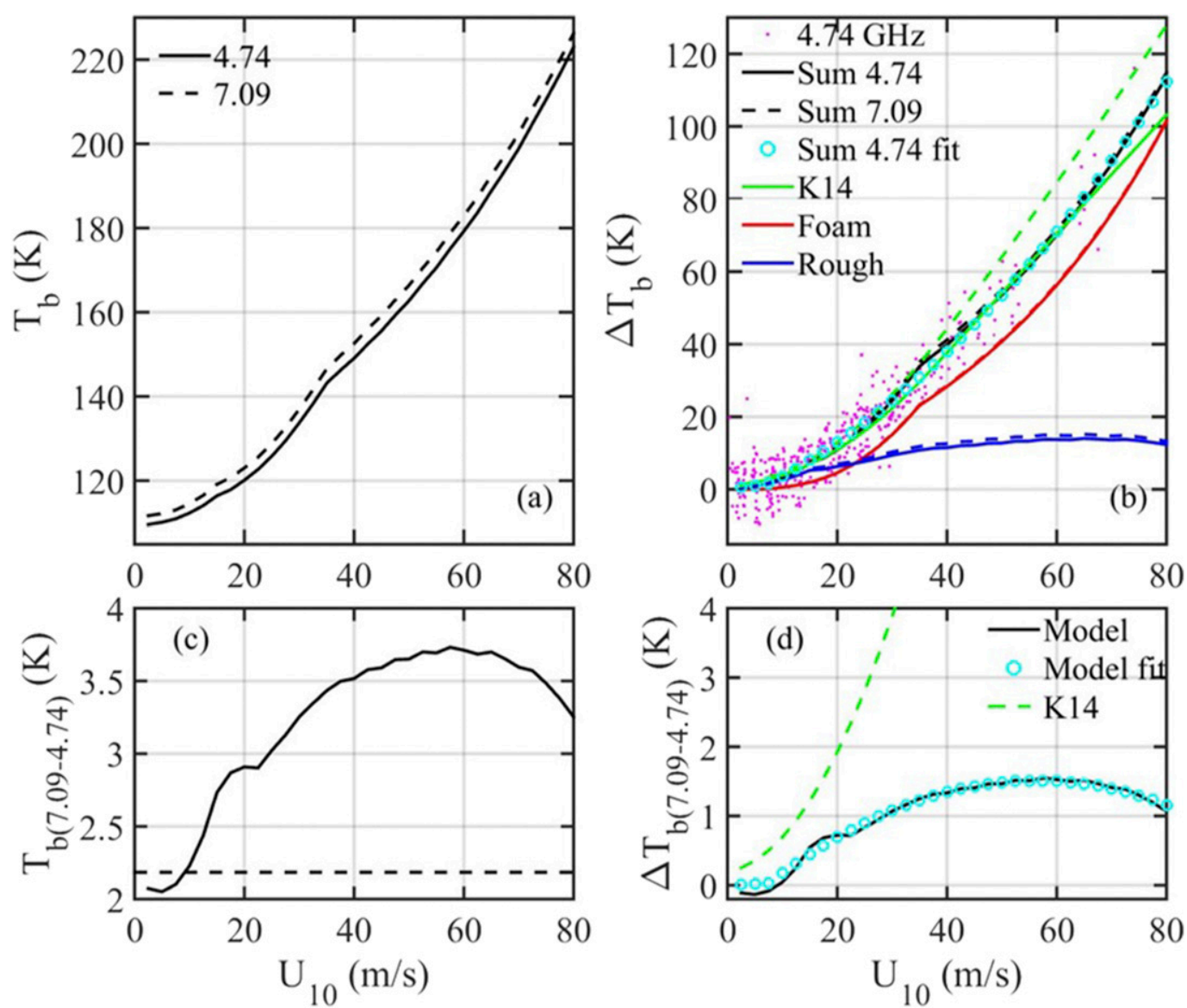

FIG. 5. (a) The thermal emission model computation of SFMR $T_{B}$ for 4.74 (solid lines) and 7.09 (dashed lines) $\mathrm{GHz}$ using $C_{10 B}$. (b) The computed sum, foam, and roughness contributions of the 4.74- and 7.09-GHz $\Delta T_{B}$. The SFMR 4.74-GHz measurements are superimposed with dots; the results computed with the SFMR excess emissivity algorithm (K14) for 4.74 and $7.09 \mathrm{GHz}$ are shown with green solid and dashed lines, respectively. (c) The computed $T_{B}$ difference between 4.74 and $7.09 \mathrm{GHz}$. (d) The computed $\Delta T_{B}$ difference between 4.74 and $7.09 \mathrm{GHz}$ and comparison with the K14 algorithm.

Although the thermal emission model (section 2) can be applied to all six SFMR frequencies, the coarse grids for the wavenumber spectrum (128 logarithmically spaced components covering $0.013-4024 \mathrm{rad} \mathrm{m}^{-1}$ ) in the present computation code are not designed to resolve closely spaced microwave frequencies. Here, only the results for 4.74 and $7.09 \mathrm{GHz}$ are presented. The computed $T_{B}$ results using $C_{10 B}$ for the two frequencies show similar wind dependency with only small differences in the magnitude (Fig. 5a). The corresponding $\Delta T_{B}$ (sum, roughness, and foam components) are shown in Fig. 5b with solid lines for $4.74 \mathrm{GHz}$ and dashed lines for $7.09 \mathrm{GHz}$. For comparison, the calculated $\Delta T_{B}$ for 4.74 and $7.09 \mathrm{GHz}$ using the K14 SFMR excess emissivity algorithm [their (5)(7)] are displayed with green solid and dashed lines, respectively.

The modeled $T_{B}$ difference between 4.74 and $7.09 \mathrm{GHz}$ is less than $4 \mathrm{~K}$ for $U_{10}$ up to $80 \mathrm{~m} \mathrm{~s}^{-1}$ as shown in Fig. $5 \mathrm{c}$, of which $2.18 \mathrm{~K}$ is the difference of the foamless flat surface values (shown with dashed line in Fig. 5c), so the wind-induced $\Delta T_{B}$ difference between 4.74 and $7.09 \mathrm{GHz}$ is less than $2 \mathrm{~K}$ (Fig. 5d). Based on the thermal emission model computation, the excess emissivity for $4.74 \mathrm{GHz}$ and the excess emissivity sensitivity to frequency (in $\mathrm{GHz}$ ) can be expressed as

$$
\Delta e_{p 4.74}=\left\{\begin{array}{cc}
3.4005 \times 10^{-5} U_{10}^{2}+2.0975 \times 10^{-3} U_{10}-1.2400 \times 10^{-2}, & U_{10} \geq 7.5 \mathrm{~m} \mathrm{~s}^{-1} \\
6.9912 \times 10^{-4} U_{10}, & U_{10}<7.5 \mathrm{~m} \mathrm{~s}^{-1}
\end{array}\right. \text { and }
$$




$$
\frac{d \Delta e_{p}}{d f}=\left\{\begin{array}{cc}
-8.8149 \times 10^{-7} U_{10}^{2}+9.9266 \times 10^{-5} U_{10}-6.5731 \times 10^{-4}, & U_{10} \geq 7.5 \mathrm{~m} \mathrm{~s}^{-1} \\
5.0138 \times 10^{-6} U_{10}, & U_{10}<7.5 \mathrm{~m} \mathrm{~s}^{-1}
\end{array},\right.
$$

which are illustrated with cyan circles in Figs. 5b and 5d. The magnitude of $d \Delta e_{p} / d f$ is considerably less than the SFMR algorithm computation given with green dashed line in Fig. 5d. It is feasible that the frequencydependent rain effects remain in the $d \Delta e_{p} / d f$ algorithm by $\mathrm{K} 14$ (p. 2397), as inferred from their description of the data used for the analysis. ("Because of the relatively limited sample size from which the $\varepsilon-U_{\text {sfc }}$ relationship was developed, measurements in precipitating regions could not be excluded, and the impact of rain on brightness temperature was estimated to compute the SFMR surface emissivity. ... A sample of dropsondes from over 200 NOAA and AFRC flights between 2005 and 2012 was used to compare with 10-s averaged SFMR-measured emissivity at the time of dropsonde launch. At lower dropsonde wind speeds of $U_{\mathrm{sfc}}<35 \mathrm{~m} \mathrm{~s}^{-1}$, measurements for which $R_{\mathrm{sfmr}}<$ $2 \mathrm{~mm} \mathrm{~h}^{-1}$ - which is near the noise level-are retained; for speeds $35<U_{\text {sfc }}<60 \mathrm{~m} \mathrm{~s}^{-1}$, only measurements with $R_{\text {sfmr }}<10 \mathrm{~mm} \mathrm{~h}^{-1}$ are saved; however, all measurements for $U_{\text {sfc }}>60 \mathrm{~m} \mathrm{~s}^{-1}$ are included, regardless of the rain rate, since the impact of rain at high wind speeds is far less than at lower wind speeds due to the high surface emissivity.")

\section{b. Foam and roughness contributions}

As illustrated in Figs. 4b and 5b, for the SFMR data the roughness contribution is the dominant component for wind speeds up to about $22 \mathrm{~m} \mathrm{~s}^{-1}$. For higher winds, the foam contribution then exceeds the roughness contribution. This result reflects the different responses of the surface roughness and whitecaps to wind forcing.

The wind dependency of the surface roughness can be expressed as the power function of the roughness spectral components: $B(k)=A(k)\left(u_{*} / c\right)^{a(k)}$, where $B$ is the dimensionless spectrum, $k$ is wavenumber, $c$ is the corresponding phase speed connected to $k$ by the surface wave dispersion relationship, and $A$ and $a$ are scalar parameters (e.g., Hwang et al. 2013; Hwang and Fois 2015). For the wavenumber components near the C-band EM wavenumber $\left(99 \mathrm{rad} \mathrm{m}^{-1}\right.$ for $4.74 \mathrm{GHz}$ and $148 \mathrm{rad} \mathrm{m}^{-1}$ for $\left.7.09 \mathrm{GHz}\right), a$ is close to 1 for $U_{10}<$ $\sim 17 \mathrm{~m} \mathrm{~s}^{-1}$ and about 0.75 for higher winds [Fig. 7 in Hwang and Fois 2015; Fig. 4 and (7) in Hwang et al. 2013]. As illustrated in Fig. 1 of Johnson and Zhang (1999), the weighting function $g_{p}$ in (4) has its peak at the EM wavenumber and drops off sharply toward both lower- and higher-frequency sides.
The wind dependency of the foam contribution is related to the whitecap fraction, which is proportional to about $u_{*}^{3}$ and $u_{*}^{2.5}$ for $U_{10}$ below and above $17 \mathrm{~m} \mathrm{~s}^{-1}$ $\left(u_{*}=0.4 \mathrm{~m} \mathrm{~s}^{-1}\right)$, respectively [Fig. $3 \mathrm{c}$ and (6)]. The whitecap coverage is usually a small fraction of the ocean surface; for example, at 10 and $20 \mathrm{~m} \mathrm{~s}^{-1}$ wind speeds, the whitecap coverages are $0.7 \%$ and $5.6 \%$, respectively. However, the nonlinear response on wind forcing (with stronger-than-quadratic dependence on the wind friction velocity) eventually leads the foam contribution to exceed the quasi-linear roughness contribution.

\section{c. Drag coefficient and whitecap fraction}

The results shown in Fig. 3 may seem to contradict some of the conclusions from previous studies. For example, several other studies suggest that the friction velocity should decrease once getting into the hurricane wind regime, while the present analysis suggests that it continues to increase (Fig. 3b). The $C_{10 A}$ formula is a typical example of the nonmonotonic wind stress dependence on wind speed (dotted curves in Fig. 3). As illustrated in Fig. 4, the brightness temperature computation using such nonmonotonic wind stress dependence on wind speed deviates from the SFMR observations considerably (dotted curve in Fig. 4a).

Also, the results of Holthuijsen et al. (2012) indicate that the whitecap coverage should remain steady above $\sim 40 \mathrm{~ms}^{-1}$, while the present analysis again suggests a continued increase in whitecap coverage. If the whitecap coverage indeed reaches some saturation level at $U_{10}$ about $40 \mathrm{~m} \mathrm{~s}^{-1}$, the foam contribution will also plateau at about $40 \mathrm{~m} \mathrm{~s}^{-1}$, leaving a big gap between the SFMR observations and thermal emission model computation (Figs. 4b, 5b).

In recent years, there have been many new reports of high-wind remote sensing analyses (e.g., Katzberg and Dunion 2009; Meissner and Wentz 2009; Yueh et al. 2010, 2013, 2016; Gleason 2013; Katzberg et al. 2013; K14; Meissner et al. 2014, 2017; Reul et al. 2016; Stoffelen et al. 2017; Gleason et al. 2018) providing invaluable information for refining the modeling of oceanographic parameters (roughness spectrum, whitecap coverage, and wind stress dependence on wind speed) relevant to remote sensing computations such as radar cross sections and radiometer brightness temperatures. The SFMR dataset (K14), being the only one covering wind speeds beyond $45 \mathrm{~m} \mathrm{~s}^{-1}$, is especially crucial for refining the formulation of the drag coefficient and related oceanographic parameters in very high winds. 

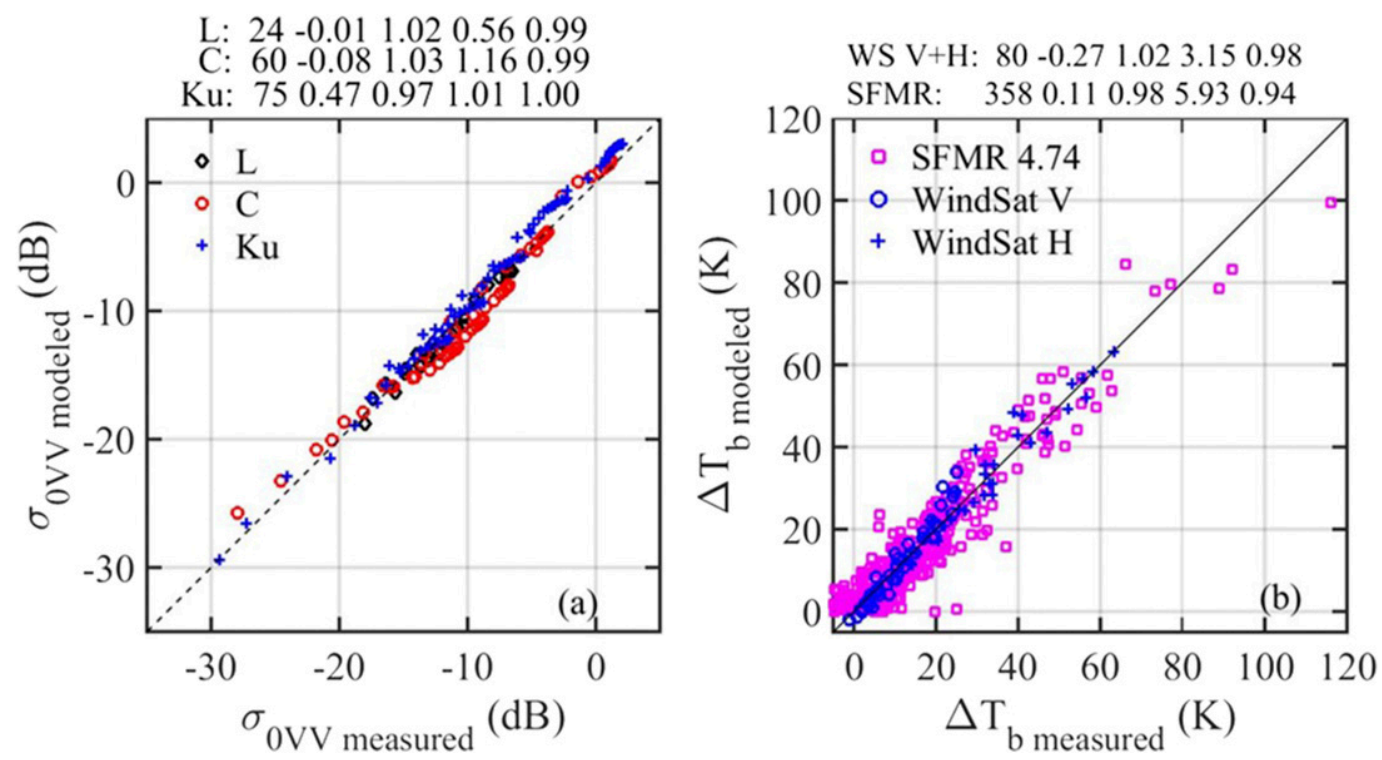

FIG. 6. Comparison of the modeled and measured radar cross sections and radiometer brightness temperature with the wind stress defined by $C_{10 B}$ in (8). (a) L-, C-, and Ku-band VV NRCS. (b) SFMR and WindSat $T_{B}$. The statistics of the number of data population, the bias, the slope of linear regression, the RMS difference, and the correlation coefficient are listed at the top of the figures.

The theories for microwave scattering and emission are well developed, and they place stringent restraints on the analysis outcome. In particular, for the models to produce good agreement of the radar cross section and radiometer brightness temperature computations in multiple frequencies with field measurements, it requires that the roughness spectrum and the whitecap fraction models as well as their driving force (wind stress represented by the drag coefficient) be reasonably accurate. The revised drag coefficient has been applied to the high-wind data from active and passive microwave sensors discussed in the last paragraph. Very good agreement is achieved between the analytical results (from NRCS and $T_{B}$ models) and field measurements (Hwang 2018, manuscript submitted to IEEE Trans. Geosci. Remote Sens.). Figure 6 shows examples of the comparison of modeled and measured microwave radar scattering and radiometer brightness temperature in calm to TC wind conditions; the drag coefficient $C_{10 B}$ in (8) is used to compute the wind stress input for the roughness spectrum and whitecap fraction. These model-measurement comparison results offer some confidence on the robustness of the revised drag coefficient formulation.

\section{Summary}

Surface waves constitute the ocean surface roughness, which significantly influences the emission and scattering of EM waves at the air-sea interface. In high winds, air entrained by wave breaking modifies the dielectric properties of the surface layer. Presently, the whitecap coverage is the most convenient observable of the surface wave breaking process. Both surface roughness and wave breaking (whitecaps) are driven by the surface wind stress; therefore, their quantification using the wind speed input relies on the drag coefficient formulation.

Microwave signals from the ocean surface contain the key information of the ocean surface roughness and whitecap coverage. The surface roughness spectrum has been refined by taking advantage of the Bragg resonance mechanism dominating the vertical transmit, vertical receive (VV) scatterometer backscattering (Hwang et al. 2013; Hwang and Fois 2015) and the low-pass-filtered mean-square slope observations using the GPS reflectometry technique inside hurricanes (Hwang and Fan 2018).

In this paper, the extensive SFMR dataset collected in 370 hurricane reconnaissance and research missions (K14) are used for refining the drag coefficient and whitecap coverage formulations. It is found that $C_{10} \sim U_{10}^{-1}$, $u_{*} \sim U_{10}^{0.5}$, and $W_{c} \sim U_{10}^{1.25}$ in very high winds $\left(U_{10}\right.$ greater than $35 \mathrm{~m} \mathrm{~s}^{-1}$ ). The brightness temperature computation using the surface roughness and whitecap coverage based on the revised drag coefficient formulation yields excellent agreement with the SFMR measurements.

Acknowledgments. This work is sponsored by the Office of Naval Research (Grant N0001416WX00044). Comments and suggestions from two reviewers have 
greatly enhanced the quality and clarity of the presentation. Datasets used in this analysis are given in the references cited. The processing codes and data segments can also be obtained by contacting the corresponding author. U.S. Naval Research Laboratory Publication Number JA/7260-18-0139.

\section{REFERENCES}

Anguelova, M. D., 2008: Complex dielectric constant of sea foam at microwave frequencies. J. Geophys. Res., 113, C08001, https:// doi.org/10.1029/2007JC004212.

Bell, M. M., M. T. Montgomery, and K. A. Emanuel, 2012: Air-sea enthalpy and momentum exchange at major hurricane wind speeds observed during CBLAST. J. Atmos. Sci., 69, 31973122, https://doi.org/10.1175/JAS-D-11-0276.1.

Brumer, S. E., C. J. Zappa, I. M. Brooks, H. Tamura, S. M. Brown, B. W. Blomquist, C. W. Fairall, and A. Cifuentes-Lorenzen, 2017: Whitecap coverage dependence on wind and wave statistics as observed during SO GasEx and HiWinGS. J. Phys. Oceanogr., 47, 2211-2235, https://doi.org/10.1175/JPO-D-17-0005.1.

Callaghan, A., G. de Leeuw, L. Cohen, and C. D. O'Dowd, 2008: Relationship of oceanic whitecap coverage to wind speed and wind history. Geophys. Res. Lett., 35, L23609, https://doi.org/ 10.1029/2008GL036165.

Felizardo, F. C., and W. K. Melville, 1995: Correlations between ambient noise and the ocean surface wave field. J. Phys. Oceanogr., 25, 513-532, https://doi.org/10.1175/1520-0485(1995)025<0513: CBANAT $>2.0 . \mathrm{CO} ; 2$.

Gleason, S., 2013: Space-based GNSS scatterometry: Ocean wind sensing using an empirically calibrated model. IEEE Trans. Geosci. Remote Sens., 51, 4853-4863, https://doi.org/10.1109/ TGRS.2012.2230401.

_, V. U. Zavorotny, D. M. Akos, S. Hrbek, I. PopStefanija, E. J. Walsh, D. Masters, and M. S. Grant, 2018: Study of surface wind and mean square slope correlation in Hurricane Ike with multiple sensors. IEEE J. Sel. Top. Appl. Earth Obs. Remote Sens., 11, 1975-1988, https://doi.org/10.1109/JSTARS.2018.2827045.

Holthuijsen, L. H., M. D. Powell, and J. D. Pietrzak, 2012: Wind and waves in extreme hurricanes. J. Geophys. Res., 117, C09003, https://doi.org/10.1029/2012JC007983.

Hwang, P. A., 2011: A note on the ocean surface roughness spectrum. J. Atmos. Oceanic Technol., 28, 436-443, https://doi.org/ 10.1175/2010JTECHO812.1

_ 2012: Foam and roughness effects on passive microwave remote sensing of the ocean. IEEE Trans. Geosci. Remote Sens. 50, 2978-2985, https://doi.org/10.1109/TGRS.2011.2177666.

_ and M. A. Sletten, 2008: Energy dissipation of windgenerated waves and whitecap coverage. J. Geophys. Res. 113, C02012, https://doi.org/10.1029/2007JC004277; Corrigendum, 114, C02015, https://doi.org/10.1029/2008JC005244.

—, and F. Fois, 2015: Surface roughness and breaking wave properties retrieved from polarimetric microwave radar backscattering. J. Geophys. Res. Oceans, 120, 3640-3657, https://doi.org/ 10.1002/2015JC010782.

_ _ and Y. Fan, 2017: Effective fetch and duration of tropical cyclone wind fields estimated from simultaneous wind and wave measurements: Surface wave and air-sea exchange computation. J. Phys. Oceanogr., 47, 447-470, https://doi.org/ 10.1175/JPO-D-16-0180.1.

_ and _ 2018: Low-frequency mean square slopes and dominant wave spectral properties: Toward tropical cyclone remote sensing. IEEE Trans. Geosci. Remote Sens., https://doi.org/ 10.1109/TGRS.2018.2850969, in press.

—, D. M. Burrage, D. W. Wang, and J. C. Wesson, 2013: Ocean surface roughness spectrum in high wind condition for microwave backscatter and emission computations. J. Atmos. Oceanic Technol., 30, 2168-2188, https://doi.org/10.1175/ JTECH-D-12-00239.1.

Jarosz, E., D. A. Mitchell, D. W. Wang, and W. J. Teague, 2007: Bottom-up determination of air-sea momentum exchange under a major tropical cyclone. Science, 315, 1707-1709, https://doi.org/10.1126/science.1136466.

Johnson, J. T., and M. Zhang, 1999: Theoretical study of the small slope approximation for ocean polarimetric thermal emission. IEEE Trans. Geosci. Remote Sens., 37, 2305-2316, https://doi.org/ $10.1109 / 36.789627$.

Katzberg, S. J., and J. Dunion, 2009: Comparison of reflected GPS wind speed retrievals with dropsondes in tropical cyclones. Geophys. Res. Lett., 36, L17602, https://doi.org/10.1029/ 2009GL039512.

, - - and G. G. Ganoe, 2013: The use of reflected GPS signals to retrieve ocean surface wind speeds in tropical cyclones. Radio Sci., 48, 371-387, https://doi.org/10.1002/rds.20042.

Klein, L., and C. Swift, 1977: An improved model for the dielectric constant of sea water at microwave frequencies. IEEE Trans. Antennas Propag., 25, 104-111, https://doi.org/10.1109/ TAP.1977.1141539.

Klotz, B. W., and E. W. Uhlhorn, 2014: Improved Stepped Frequency Microwave Radiometer tropical cyclone surface winds in heavy precipitation. J. Atmos. Oceanic Technol., 31, 23922408, https://doi.org/10.1175/JTECH-D-14-00028.1.

Lafon, C., J. Piazzola, P. Forget, O. Le Calve, and S. Despiau, 2004: Analysis of the variations of the whitecap fraction as measured in a coastal zone. Bound.-Layer Meteor., 111, 339-360, https:// doi.org/10.1023/B:BOUN.0000016490.83880.63.

- — - - - and S. Despiau, 2007: Whitecap coverage in coastal environment for steady and unsteady wave field conditions. J. Mar. Syst., 66, 38-46, https://doi.org/10.1016/ j.jmarsys.2006.02.013.

Meissner, T., and F. J. Wentz, 2004: The complex dielectric constant of pure and sea water from microwave satellite observations. IEEE Trans. Geosci. Remote Sens., 42, 1836-1849, https://doi.org/10.1109/TGRS.2004.831888.

_ and _ - 2009: Wind-vector retrievals under rain with passive satellite microwave radiometers. IEEE Trans. Geosci. Remote Sens., 47, 3065-3083, https://doi.org/10.1109/ TGRS.2009.2027012.

_, , and L. Ricciardulli, 2014: The emission and scattering of L-band microwave radiation from rough ocean surfaces and wind speed measurements from the Aquarius sensor. J. Geophys. Res. Oceans, 119, 6499-6522, https://doi.org/ 10.1002/2014JC009837.

—_ L. Ricciardulli, and F. J. Wentz, 2017: Capability of the SMAP mission to measure ocean surface winds in storms. Bull. Amer. Meteor. Soc., 98,1660-1677, https://doi.org/10.1175/ BAMS-D-16-0052.1.

Monahan, E. C., 1971: Oceanic whitecaps. J. Phys. Oceanogr., 1, 139-144, https://doi.org/10.1175/1520-0485(1971)001<0139: OW $>2.0 . \mathrm{CO} ; 2$.

Plant, W. J., 1990: Bragg scattering of electromagnetic waves from the air/sea interface. Remote Sensing, G. L. Geernaert and W. L. Plant, Eds., Vol. II, Surface Waves and Fluxes, Kluwer, 41-108.

Powell, M. D., 2006: Drag coefficient distribution and wind speed dependence in tropical cyclones: Final report. NOAA Rep., 26 pp. 
P. J. Vickery, and T. A. Reinhold, 2003: Reduced drag coefficient for high wind speeds in tropical cyclones. Nature, $\mathbf{4 2 2}$, 279-283, https://doi.org/10.1038/nature01481.

Reul, N., and B. Chapron, 2003: A model of sea-foam thickness distribution for passive microwave remote sensing applications. J. Geophys. Res., 108, C103321, https://doi.org/10.1029/ 2003JC001887.

, - - E. Zabolotskikh, C. Donlon, Y. Quilfen, S. Guimbard, and J. F. Piolle, 2016: A revised L-band radio-brightness sensitivity to extreme winds under tropical cyclones: The five year SMOS-storm database. Remote Sens. Environ., 180, 274 291, https://doi.org/10.1016/j.rse.2016.03.011.

Ross, D. B., and V. Cardone, 1974: Observations of oceanic whitecaps and their relation to remote measurements of surface wind speed. J. Geophys. Res., 79, 444-452, https://doi.org/ 10.1029/JC079i003p00444.

Stoffelen, A., J. A. Verspeek, J. Vogelzang, and A. Verhoef, 2017: The CMOD7 geophysical model function for ASCAT and ERS wind retrievals. IEEE J. Sel. Top. Appl. Earth Obs. Remote Sens., 10, 2123-2134, https://doi.org/10.1109/ JSTARS.2017.2681806.

Stogryn, A., 1972: The emissivity of sea foam at microwave frequencies. J. Geophys. Res., 77, 1658-1666, https://doi.org/ 10.1029/JC077i009p01658.

Sugihara, Y., H. Tsumori, T. Ohga, H. Yoshioka, and S. Serizawa, 2007: Variation of whitecap coverage with wave-field conditions. J. Mar. Syst., 66, 47-60, https://doi.org/10.1016/j.jmarsys.2006.01.014.

Toba, Y., and M. Chaen, 1973: Quantitative expression of the breaking of wind waves on the sea surface. Rec. Oceanogr. Works Japan, 12, 1-11.

Uhlhorn, E. W., and P. G. Black, 2003: Verification of remotely sensed sea surface winds in hurricanes. J. Atmos. Oceanic Technol., 20, 99-116, https://doi.org/10.1175/1520-0426(2003)020<0099: VORSSS $>2.0 . \mathrm{CO} ; 2$. $\longrightarrow,-$ J. L. Franklin, M. Goodberlet, J. Carswell, and A. S. Goldstein, 2007: Hurricane surface wind measurements from an operational stepped frequency microwave radiometer. Mon. Wea. Rev., 135, 3070-3085, https://doi.org/10.1175/MWR3454.1.

Wright, J., 1966: Backscattering from capillary waves with application to sea clutter. IEEE Trans. Antennas Propag., 14, 749754, https://doi.org/10.1109/TAP.1966.1138799.

_ 1968: A new model for sea clutter. IEEE Trans. Antennas Propag., 16, 217-223, https://doi.org/10.1109/TAP.1968.1139147.

Xu, D., X. Liu, and D. Yu, 2000: Probability of wave breaking and whitecap coverage in a fetch-limited sea. J. Geophys. Res., $\mathbf{1 0 5}$, 14 253-14259, https://doi.org/10.1029/2000JC900040.

Yueh, S. H., R. Kwok, F. K. Li, S. V. Nghiem, W. J. Wilson, and J. A. Kong, 1994a: Polarimetric passive remote sensing of ocean wind vectors. Radio Sci., 29, 799-814, https://doi.org/ 10.1029/94RS00450.

—,- , and S. V. Nghiem, 1994b: Polarimetric scattering and emission properties of targets with reflection symmetry. Radio Sci., 29, 1409-1420, https://doi.org/10.1029/94RS02228.

— S. J. Dinardo, A. G. Fore, and F. K. Li, 2010: Passive and active L-band microwave observations and modeling of ocean surface winds. IEEE Trans. Geosci. Remote Sens., 48, 3087 3100, https://doi.org/10.1109/TGRS.2010.2045002.

— W. Tang, A. G. Fore, G. Neumann, A. Hayashi, A. Freedman, J. Chaubell, and G. S. E. Lagerloef, 2013: L-band passive and active microwave geophysical model functions of ocean surface winds and applications to Aquarius retrieval. IEEE Trans. Geosci. Remote Sens., 51, 4619-4632, https://doi.org/ 10.1109/TGRS.2013.2266915.

—, A. G. Fore, W. Tang, A. Hayashi, B. Stiles, N. Reul, Y. Weng, and F. Zhang, 2016: SMAP L-band passive microwave observations of ocean surface wind during severe storms. IEEE Trans. Geosci. Remote Sens., 54, 7339-7350, https://doi.org/ 10.1109/TGRS.2016.2600239. 